\title{
New Families of Solitary Pattern Solutions of the Nonlinear Dispersive $K(n, m, k)$ Equations
}

\author{
Mustafa Inc \\ Department of Mathematics, Firat University, Elazig 23119, Turkiye \\ Reprint requests to Dr. M. I.; E-mail:minc@ firat.edu.tr
}

Z. Naturforsch. 59a, 275 - 280 (2004); received January 8, 2004

In this paper we shall derive new solitary pattern solutions of the nonlinear dispersive KdV equation (shortly called $K(n, m, k)$ ) by use of simple methods. The properties of these $K(n, m, k)$ equations are shown in figures.

Key words: Solitary Pattern; Solitary Wave Solution; Nonlinear Dispersion.

\section{Introduction}

Travelling wave solutions are important in nonlinear science. These solutions describe phenomena such as vibrations, solitons and propagation with finite speed. In recent years, direct search for exact solutions of nonlinear partial differential equations (PDEs) has become more and more attractive, partly due to the availability of computer systems like Maple or Mathematica, which allow to perform complicated and tedious algebraic calculations and to find new exact solutions of PDEs [1-4].

In 1993 Rosenau and Hyman [5] introduced a class of PDEs, i.e.

$$
\begin{gathered}
K(m, n): u_{t}+a\left(u^{m}\right)_{x}+\left(u^{n}\right)_{x x x}=0 \\
m>1,1 \leq n \leq 3
\end{gathered}
$$

which are generalizations of the Korteweg-de Vries $(\mathrm{KdV})$ equation. For certain values of $m$ and $n$, the $K(m, n)$ equation yields solitary waves which are compactly supported. For $m=n$ these solitary waves, the so-called compactons, take the particularly simple form

$$
\begin{gathered}
u=\left\{\frac{2 n \lambda}{n+1} \cos \left[\frac{n-1}{4 n}(x-\lambda t)\right]\right\}^{2 /(n-1)}, \\
\text { when }|x-\lambda t| \leq \frac{2 n \pi}{n-1} \text { and } \\
u=0, \text { otherwise. }
\end{gathered}
$$

For $a<0$ one obtains solitary patterns having cusps or infinite slopes [6]. In (1), if $a=1$, then this equation has a focusing $(+)$ branch, and if $a=-1$, then this equation has a defocusing $(-)$ branch. It is shown that the focusing branches of these equations exhibit compactons that are solitons with infinite wavelength, however the defocusing branches support solitary pattern solutions with infinite slopes or cusps. More works on compactons and solitary pattern solutions of this type of equations can be found in [7-14].

We consist the following nonlinear dispersive $K(n, m), K(n, m, k)$ and $K(n, m, k, l)$ equations:

$$
\begin{gathered}
u_{t}+a\left(u^{n}\right)_{x}+b\left(u^{m}\right)_{x x x}=0 \\
u_{t}+a\left(u^{n}\right)_{x}+b\left(u^{m}\right)_{x x x}+c\left(u^{k}\right)_{y y y}=0 \\
u_{t}+a\left(u^{n}\right)_{x}+b\left(u^{m}\right)_{x x x}+c\left(u^{k}\right)_{y y y}+r\left(u^{l}\right)_{z z z}=0
\end{gathered}
$$

where $a, b, c, m, n, k, l$ and $r>0$ are constants. The aim of this paper is to investigate other new solitary pattern solutions of (4), which is called the $K(n, m, k)$ equation. In addition, we can also obtain new solitary pattern solutions of (3) and (5) in a similar manner.

The rest of this paper is organized as follows: In Sect. 2 we give several general solitary pattern solutions and some solitary and singular solitary wave solutions of (4). In the last Section some conclusions are given.

\section{General Formulas of Solitary Pattern Solutions of Eq. (4)}

We first make the travelling wave transformation

$$
u(x, y, t)=U(\xi), \xi=x+y-\lambda t
$$


where $\lambda$ is a constant. Then (4) reduces to

$$
-\lambda U^{\prime}+a\left(U^{n}\right)^{\prime}+b\left(U^{m}\right)^{\prime \prime \prime}+c\left(U^{k}\right)^{\prime \prime \prime}=0 .
$$

Integrating (7) once and setting the integration constant to zero, we have

$$
-\lambda U+a U^{n}+b\left(U^{m}\right)^{\prime \prime}+c\left(U^{k}\right)^{\prime \prime}=0 .
$$

We assume that the general solitary pattern solutions have three different forms [8-11]:

\section{Type A:}

$$
u(x, y, t)=U(\xi)=P \sinh ^{\beta}(R \xi),
$$

\section{Type B:}

$$
u(x, y, t)=U(\xi)=P \cosh ^{\beta}(R \xi),
$$

\section{Type C:}

$$
u(x, y, t)=U(\xi)=P(\cosh (R \xi)+\sinh (R \xi))^{\beta}
$$

where $P, R$ and $\beta$ are constants to be determined later.

Substituting (9) into (8), with the aid of Mathematica we easily have

$$
\begin{aligned}
& m=n, \beta=\frac{2}{k-1}, P^{k-1}=-\frac{2 \lambda b m^{2}}{a c k(k+1)}, \\
& R=\sqrt{-\frac{a}{b}} \frac{k-1}{2 m}, a b<0, \\
& n=k, \beta=\frac{2}{k-n}, P^{k-n}=\frac{2 k}{k+n}, \\
& R=\sqrt{-\frac{a}{c}} \frac{k-n}{2 k}, a c<0, \\
& m=1, \beta=-\frac{2}{n-1}, P^{n-1}=-\frac{8 m b(1-2 m-n)}{a(n-1)^{4}}, \\
& R=\sqrt{\frac{\lambda}{b}} \frac{n-1}{2}, \lambda b>0, \\
& n=k, \beta=\frac{2}{m-1}, P^{m-1}=-\frac{\lambda c(m-1)}{m a(m+1)}, \\
& R=\sqrt{-\frac{a}{c}} \frac{m-1}{2 k}, a c<0, \\
& k \beta-1=0, a\left(b m^{2}+k^{2}\right)<0 . \\
& m=n=k, \beta=\frac{1}{k}, R=k \sqrt{-\frac{1}{a\left(b m^{2}+k^{2}\right)}}, \\
& n=1
\end{aligned}
$$

In view of $(12)-(16)$, we have the following conclusions:

\subsection{Solitary Pattern and Solitary Wave Solutions of Eq. (4)}

\section{Type A:}

Case 1. When $m=n \neq k$, the exact solution of the $K(m, m, k)$ equations is

$$
\begin{aligned}
u(x, y, t)=\{ & -\frac{2 \lambda b m^{2}}{a c k(k+1)} \\
& \left.\cdot \sinh ^{2}\left[\sqrt{-\frac{a}{b}} \frac{k-1}{2 m}(x+y-\lambda t)\right]\right\}^{1 /(k-1)} .
\end{aligned}
$$
to solutions of the $K(n, n)$ equation by Wazwaz [13] and Yan $[10,11]$. For $m=n<k$, it is easily seen that (17) becomes

$$
\begin{aligned}
u(x, y, t)=\{ & -\frac{a c k(k+1)}{2 \lambda b m^{2}} \\
& \left.\cdot \operatorname{csch}^{2}\left[\sqrt{-\frac{a}{b}} \frac{k-1}{2 m}(x+y-\lambda t)\right]\right\}^{1 /(1-k)},
\end{aligned}
$$

which is the bell-shaped solitary wave solution.

Case 2. When $n=k \neq m$, the exact solution of the $K(n, m, n)$ equations is given by

$$
\begin{aligned}
u(x, y, t)=\{ & \frac{2 k}{k+n} \\
& \left.\cdot \sinh ^{2}\left[\sqrt{-\frac{a}{c} \frac{k-n}{2 k}}(x+y-\lambda t)\right]\right\}^{1 /(k-n)} .
\end{aligned}
$$

For $n=k<m$ it is easy to see that (19) becomes the solitary wave solution

$$
\begin{aligned}
u(x, y, t)=\{ & \frac{k+n}{2 k} \\
& \left.\cdot \operatorname{csch}^{2}\left[\sqrt{-\frac{a}{c}} \frac{k-n}{2 k}(x+y-\lambda t)\right]\right\}^{1 /(n-k)} .
\end{aligned}
$$

Case 3. When $m=1, n \neq 1$, the exact solution of the $K(n, 1, k)$ equations is

$$
\begin{aligned}
u(x, y, t)=\{ & -\frac{8 b m(1-2 m-n)}{a(n-1)^{4}} \\
& \left.\cdot \sinh ^{2}\left[\sqrt{\frac{\lambda}{b}} \frac{n-1}{2}(x+y-\lambda t)\right]\right\}^{1 /(n-1)} .
\end{aligned}
$$


For $m=1, n \neq 1$ it is easy to see that (21) becomes the solitary wave solution

$$
\begin{aligned}
u(x, y, t)=\{ & -\frac{a(n-1)^{4}}{8 b m(1-2 m-n)} \\
& \left.\cdot \operatorname{csch}^{2}\left[\sqrt{\frac{\lambda}{b}} \frac{n-1}{2}(x+y-\lambda t)\right]\right\}^{1 /(1-n)} .
\end{aligned}
$$

Case 4. When $n=k \neq m$, the exact solution of the $K(k, m, k)$ equations is

$$
\begin{aligned}
u(x, y, t)=\{ & -\frac{c \lambda(m-1)}{m a(m+1)} \\
& \left.\cdot \sinh ^{2}\left[\sqrt{-\frac{a}{c}} \frac{m-1}{2 k}(x+y-\lambda t)\right]\right\}^{\frac{1}{m-1}} .
\end{aligned}
$$

For $n=k \neq m$ it is easy to see that (23) becomes the solitary wave solution

$$
\begin{aligned}
u(x, y, t)=\{ & -\frac{m a(m+1)}{c \lambda(m-1)} \\
& \left.\cdot \operatorname{csch}^{2}\left[\sqrt{-\frac{a}{c}} \frac{m-1}{2 k}(x+y-\lambda t)\right]\right\}^{\frac{1}{1-m}} .
\end{aligned}
$$

Case 5. When $m=n=k$, the exact solution of the $K(n, n, n)$ equations is

$$
u(x, y, t)=P\left\{\sinh \left[k \sqrt{-\frac{1}{a\left(b m^{2}+k^{2}\right)}}(x+y-\lambda t)\right]\right\}^{\frac{1}{k}} .
$$

Remark 2. When $m=n=k<0$ it is easy to see that (25) becomes

$$
\begin{array}{r}
u(x, y, t)=P\left\{\operatorname { c s c h } \left[k \sqrt{-\frac{1}{a\left(b m^{2}+k^{2}\right)}}\right.\right. \\
\cdot(x+y-\lambda t)]\}^{-\frac{1}{k}},
\end{array}
$$

which is the singular solitary wave solution of (4). The solution develops a singularity at a finite point, i.e., for any fixed $t=t_{0}$ there exists a point $x=x_{0}$ where the solution blows-up [15].

Type B: Similar to Type A, substituting (10) into (8) we can obtain other solitary patterns and solitary wave solutions of (4).

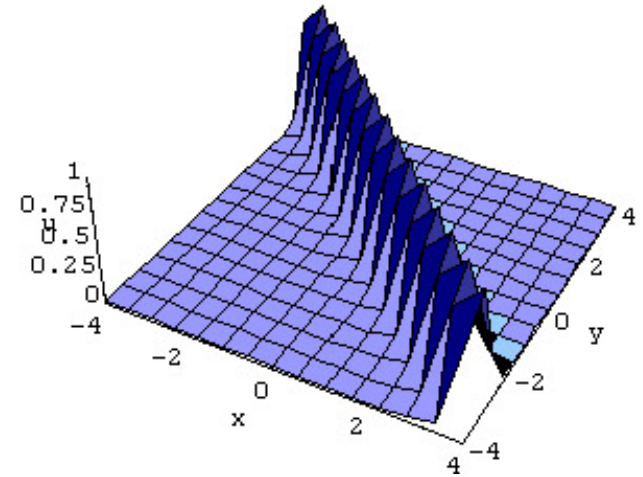

Fig. 1. The surface shows the bell-shaped solitary wave solution of (18) for $-4 \leq x, y \leq 4$, where $a=4, c=t=1, \lambda=0.5$ and $b=k=-2$.

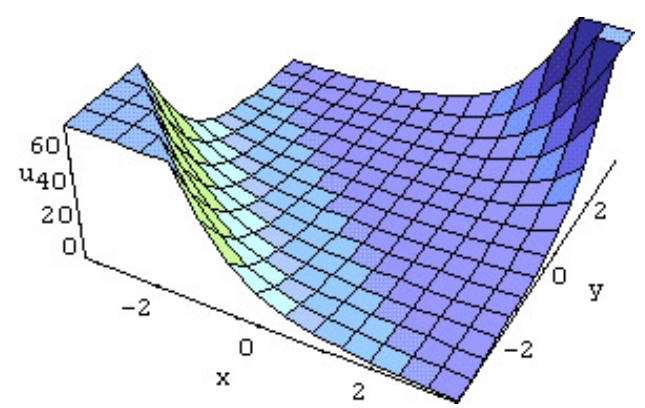

Fig. 2. The surface shows the solitary pattern solution of (19) for $-3.5 \leq x, y \leq 3.5$, where $a=4, c=t=1, \lambda=0.5, n=1$ and $k=2$.

Case 6. When $m=n \neq k$, then the exact solution of the $K(m, m, k)$ equations is given by

$$
u(x, y, t)=\left\{\frac{2 \lambda b m^{2}}{a c k(k+1)}\right.
$$

$$
\left.\cdot \cosh ^{2}\left[\sqrt{-\frac{a}{b}} \frac{k-1}{2 m}(x+y-\lambda t)\right]\right\}^{1 /(k-1)} .
$$

Remark 3. We know that (27) is a solitary pattern solution having infinite slopes or cusps. For $m=n>k$ we know that (27) is a solitary pattern solution that is similar to solutions of the $m K(m, n, k)$ equation by Yan [11]. For $m=n<k$, it easily seen that (27) becomes

$$
\begin{aligned}
u(x, y, t)=\{ & \frac{a c k(k+1)}{2 \lambda b m^{2}} \\
& \left.\cdot \operatorname{sech}^{2}\left[\sqrt{-\frac{a}{b}} \frac{k-1}{2 m}(x+y-\lambda t)\right]\right\}^{1 /(1-k)}
\end{aligned}
$$

which is the solitary wave solution. 


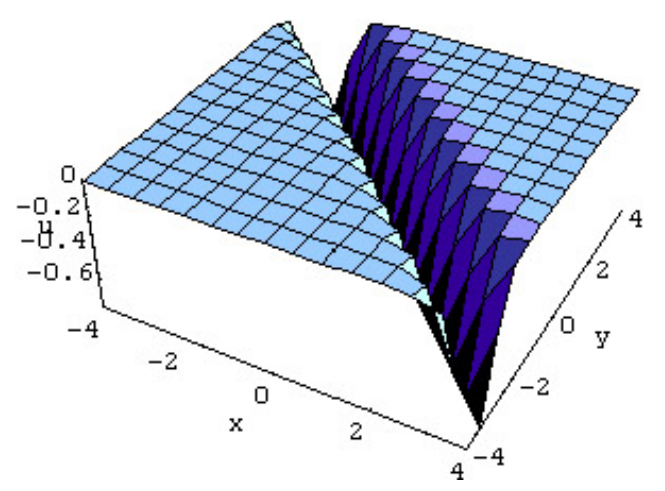

Fig. 3. The surface shows the dark-shaped solitary wave solution of (28) for $-4 \leq x, y \leq 4$, where $b=k=-2, c=m=$ $t=1, \lambda=0.5$ and $a=4$..

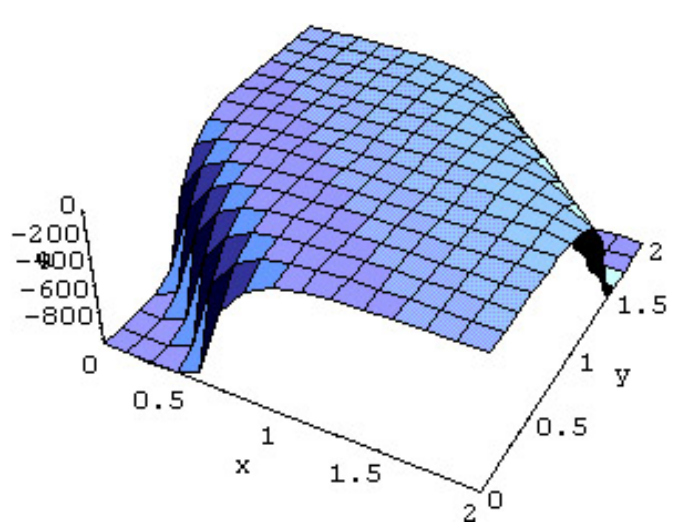

Fig. 4. The surface shows the solitary pattern solution of (29) for $0 \leq x, y \leq 2$, where $a=-4, c=t=1, \lambda=2, n=2$ and $k=-1$.

Case 7. When $n=k \neq m$, then the exact solution of the $K(n, m, n)$ equations is given by

$u(x, y, t)=\left\{\frac{2 k}{k+n}\right.$

$$
\left.\cdot \cosh ^{2}\left[\sqrt{-\frac{a}{c}} \frac{k-n}{2 k}(x+y-\lambda t)\right]\right\}^{1 /(k-n)} .
$$

For $n=k>m$ we know that (29) is a solitary pattern solution. In addition, for $n=k<m$ it is easily seen that (29) becomes the solitary wave solution

$$
\begin{aligned}
u(x, y, t)=\{ & \frac{k+n}{2 k} \\
& \left.\cdot \operatorname{sech}^{2}\left[\sqrt{-\frac{a}{c}} \frac{k-n}{2 k}(x+y-\lambda t)\right]\right\}^{1 /(n-k)} .
\end{aligned}
$$

Case 8. When $m=1, n \neq 1$, then the exact solution of $K(n, 1, k)$ equations is given by

$$
\begin{aligned}
u(x, y, t)=\{ & -\frac{8 b m(1-2 m-n)}{a(n-1)^{4}} \\
& \left.\cdot \cosh ^{2}\left[\sqrt{\frac{\lambda}{b}} \frac{n-1}{2}(x+y-\lambda t)\right]\right\}^{1 /(n-1)} .
\end{aligned}
$$

For $m=1, n \neq 1$, we know that (31) is a solitary pattern solution of (4). While when $m=1, n \neq 1<k$ it is easily seen that (31) becomes the solitary wave solution

$$
\begin{aligned}
u(x, y, t)=\{ & -\frac{a(n-1)^{4}}{8 b m(1-2 m-n)} \\
& \left.\cdot \operatorname{sech}^{2}\left[\sqrt{\frac{\lambda}{b}} \frac{n-1}{2}(x+y-\lambda t)\right]\right\}^{1 /(1-n)} .
\end{aligned}
$$

Case 9. When $n=k \neq m$, then the exact solution of the $K(k, m, k)$ equations is given by

$$
\begin{aligned}
u(x, y, t)=\{ & -\frac{c \lambda(m-1)}{m a(m+1)} \\
& \left.\cdot \cosh ^{2}\left[\sqrt{-\frac{a}{c}} \frac{m-1}{2 k}(x+y-\lambda t)\right]\right\}^{\frac{1}{m-1}} .
\end{aligned}
$$

For $n=k \neq m$ we know that (33) is a solitary pattern solution. If $n=k<m$ it is easily seen that (33) becomes the solitary wave solution

$$
\begin{aligned}
u(x, y, t)=\{ & -\frac{m a(m+1)}{c \lambda(m-1)} \\
& \left.\cdot \operatorname{sech}^{2}\left[\sqrt{-\frac{a}{c}} \frac{m-1}{2 k}(x+y-\lambda t)\right]\right\}^{\frac{1}{1-m}} .
\end{aligned}
$$

Case 10. When $m=n=k$, then the exact solution of the $K(n, n, n)$ equations is given by

$u(x, y, t)=P\left\{\cosh \left[k \sqrt{-\frac{1}{a\left(b m^{2}+k^{2}\right)}}(x+y-\lambda t)\right]\right\}^{\frac{1}{k}}$,

while when $m=n=k<0$ it is easy to see that (35) becomes

$$
\begin{gathered}
u(x, y, t)=P\left\{\operatorname { s e c h } \left[k \sqrt{-\frac{1}{a\left(b m^{2}+k^{2}\right)}}\right.\right. \\
\cdot(x+y-\lambda t)]\}^{-1 / k},
\end{gathered}
$$




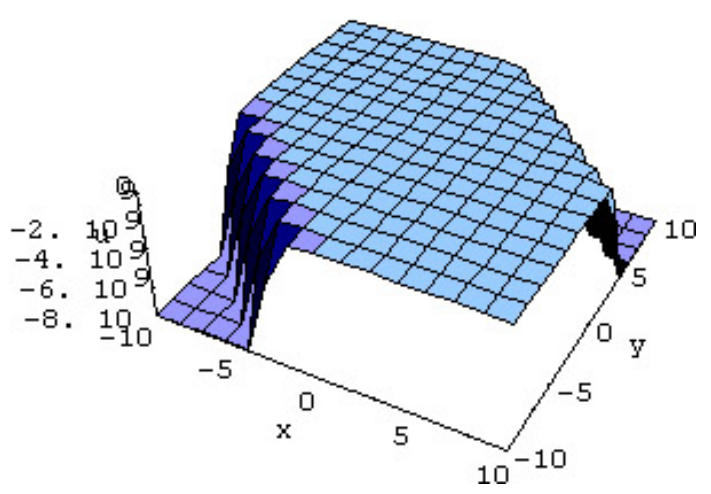

Fig. 5. The surface shows the solitary pattern solution of (42) for $-10 \leq x, y \leq 10$, where $k=2, c=t=1, \lambda=0.5$ and $a=-1$.

which is the singular solitary wave solution of (4).

Type C: Substituting (11) into (8), we obtain another solitary pattern and solitary wave solutions of (4):

$n=k, \beta=\frac{2}{k-1}, P^{k-1}=\frac{\lambda(k-1)^{2}}{2 c k(k-3)}$,

$R=\sqrt{-\frac{a(k-1)}{2 c k}}, c k<0$,

$n=k, \beta=-\frac{2}{k-m}, P^{k-m}=-\frac{b m(k-m)}{2 c k(n-k+2)}$,

$R=\sqrt{-\frac{a(k-m)}{2 c k}}, c k<0$

$k=1, \beta=-\frac{2}{n-1}, P^{n-1}=-\frac{2 c k(n-3)}{a(n-1)^{2}}$,

$R=\sqrt{-\frac{\lambda(1-n)}{2 c k}}, c k<0$

$m=k, \beta=\frac{2}{k-n}, P^{k-n}=\frac{a(k-n)^{2}}{2 c k(n-k+2)}$,

$R=\sqrt{\frac{c k}{b m}}$

$m=n=k, \beta=1, R=\sqrt{-\frac{a}{b m+c k}}$,

$\beta-1=0, a<0$.

Case 11. When $n=k \neq m$, then the exact solution of the $K(k, m, k)$ equations is given by

$$
\begin{aligned}
u=\left\{\frac { 2 c k ( k - 3 ) } { \lambda ( k - 1 ) ^ { 2 } } \left[\cosh \left(\sqrt{-\frac{a(k-1)}{2 c k}}(\xi)\right)\right.\right. \\
\left.\left.+\sinh \left(\sqrt{-\frac{a(k-1)}{2 c k}}(\xi)\right)\right]^{2}\right\}^{1 /(k-1)} .
\end{aligned}
$$

For $n=k<m$ we get the solitary wave solution

$$
\begin{aligned}
u=\left\{\frac { \lambda ( k - 1 ) ^ { 2 } } { 2 c k ( k - 3 ) } \left[\operatorname{sech}\left(\sqrt{-\frac{a(k-1)}{2 c k}}(\xi)\right)\right.\right. \\
\left.\left.\quad+\operatorname{csch}\left(\sqrt{-\frac{a(k-1)}{2 c k}}(\xi)\right)\right]^{2}\right\}^{1 /(1-k)} .
\end{aligned}
$$

Case 12. When $n=k \neq m$, then the exact solution of the $K(k, m, k)$ equations is given by

$$
\begin{aligned}
u=\{ & -\frac{2 c k(n-k+2)}{b m(k-m)} \\
& \left.\cdot[\cosh \sqrt{-A}(\xi)+\sinh \sqrt{-A}(\xi)]^{2}\right\}^{\frac{1}{k-m}},
\end{aligned}
$$

where $A=a(k-m) / 2 c k$. For $n=k<m$, we obtain the solitary wave solution

$$
\begin{aligned}
u=\{ & -\frac{b m(k-m)}{2 c k(n-k+2)} \\
& \left.\cdot[\operatorname{sech} \sqrt{-A}(\xi)+\operatorname{csch} \sqrt{-A}(\xi)]^{2}\right\}^{\frac{1}{m-k}} .
\end{aligned}
$$

Case 13. When $k=1, n \neq 1$, then the exact solution of he $K(n, 1, k)$ equations is given by

$$
\begin{array}{r}
u=\left\{-\frac{a(n-1)^{2}}{2 c k(n-3)}\left[\cosh \left(\sqrt{-\frac{\lambda(1-n)}{2 c k}}(\xi)\right)\right.\right. \\
\left.\left.+\sinh \left(\sqrt{-\frac{\lambda(1-n)}{2 c k}}(\xi)\right)\right]^{2}\right\}^{\frac{1}{n-1}} .
\end{array}
$$

For $n=k<m$ we get the solitary wave solution

$$
\begin{array}{r}
u=\left\{-\frac{2 c k(n-3)}{a(n-1)^{2}}\left[\operatorname{sech}\left(\sqrt{-\frac{\lambda(1-n)}{2 c k}}(\xi)\right)\right.\right. \\
\left.\left.+\operatorname{csch}\left(\sqrt{-\frac{\lambda(1-n)}{2 c k}}(\xi)\right)\right]^{2}\right\}^{\frac{1}{1-n}} .
\end{array}
$$

Case 14. When $m=k \neq n$, then the exact solution of the $K(n, m, m)$ equations is given by

$$
\begin{aligned}
u=\left\{\frac{2 c k(n-k+2)}{a(k-n)^{2}}\right. & {\left[\cosh \left(\sqrt{\frac{c k}{b m}}(\xi)\right)\right.} \\
+ & \left.\left.\sinh \left(\sqrt{\frac{c k}{b m}}(\xi)\right)\right]^{2}\right\}^{\frac{1}{k-n}} .
\end{aligned}
$$

For $m=k<n$ we get the solitary wave solution

$$
\begin{aligned}
u=\left\{\frac{a(k-n)^{2}}{2 c k(n-k+2)}\right. & {\left[\operatorname{sech}\left(\sqrt{\frac{c k}{b m}}(\xi)\right)\right.} \\
& \left.\left.+\operatorname{csch}\left(\sqrt{\frac{c k}{b m}}(\xi)\right)\right]^{2}\right\}^{\frac{1}{n-k}} .
\end{aligned}
$$



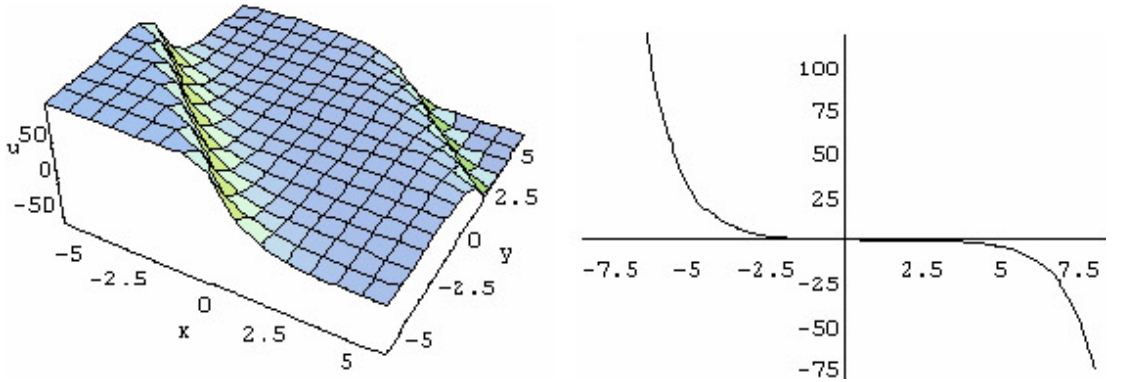

Fig. 6. The surface shows the solitary wave solution of (43) for $-6 \leq x, y \leq 6$, where $k=$ $2, c=t=1, \lambda=0.5, a=-1$.
Case 15. When $m=n=k$, then the exact solution of the $K(n, n, n)$ equations is given by

$$
\begin{aligned}
u=P\{ & \cosh \left(\sqrt{-\frac{a}{b m+c k}}(x+y-\lambda t)\right) \\
& \left.+\sinh \left(\sqrt{-\frac{a}{b m+c k}}(x+y-\lambda t)\right)\right\} .
\end{aligned}
$$

When $k=m=n<0$, it is easy to see that (50) becomes

$$
\begin{aligned}
u=P\{ & \operatorname{sech}\left(\sqrt{-\frac{a}{b m+c k}}(x+y-\lambda t)\right) \\
& \left.+\operatorname{csch}\left(\sqrt{-\frac{a}{b m+c k}}(x+y-\lambda t)\right)\right\},
\end{aligned}
$$

which is the singular solitary wave solution of (4).

Remark 4. Wazwaz [13] obtained some compacton solutions for the nonlinear dispersive $K(n, n), K(n, n, n)$ and $K(n, n, n, n)$ equations, but we present a new form of the solitary pattern solutions for nonlinear dispersive $K(n, m, k)$ equations of Type C. This type of solution

[1] E. G. Fan, Phys. Lett. A 277, 212 (2000).

[2] E. G. Fan, Phys. Lett. A 282, 18 (2001).

[3] E. G. Fan, Z. Naturforsch 56a, 312 (2001).

[4] E. G. Fan, Chaos, Solitons and Fractals 16, 819 (2003).

[5] P. Rosenau and J. M. Hyman, Phys. Rev. Lett. 70, 564 (1993).

[6] P .Rosenau, Phys. Lett. A 230, 305 (1997).

[7] P. Rosenau and S. Kamin, Physica D 8, 273 (1983).

[8] Z. Yan, Chaos, Solitons and Fractals 14, 1151 (2002).

[9] Z. Yan and G. Bluman, Comp. Phys. Commun. 149, 11 (2002). was theoretically presented by Yan [11] for the modified nonlinear dispersive $m K(m, n, k)$ equations, but he has not obtained this type of solitary pattern solutions. In this paper, the presented solutions of (4) were not found by any of the methods [13-16]. The properties of some solutions are shown in Figures 1-6.

\section{Conclusions}

In this paper we obtained several types of solitary pattern solutions of two-dimensional nonlinear dispersion $K(n, m, k)$ equations for different parameters $n, m$ and $k$. Thus, by this work we extended the previous studies on solitary pattern solutions for twodimensional nonlinear dispersion $K(n, m, k)$ equations and developed general formulas for the solutions of this type of equations with constant coefficients.

The present method is direct and efficient to obtain new solitary pattern and solitary wave solutions of (4). Our method is very easily applied to both this type of nonlinear dispersive and the modified nonlinear dispersive equations in higher dimensional spaces.

[10] Z. Yan, Chaos, Solitons and Fractals 15, 891 (2003).

[11] Z. Yan, Comp. Phys. Commun. 152, 25 (2003).

[12] M. S. Ismail and T. Taha, Math. Comput. Simulation 47, 519 (1998).

[13] A. M. Wazwaz, Appl. Math. Comput. 133, 213 (2002).

[14] A. Ludu and J. P. Draayer, Physica D 123, 82 (1998).

[15] P. A. Clarkson and E. Mansifield, Physica D 70, 70 (1993).

[16] A. M. Wazwaz, Chaos, Solitons and Fractals 13, 321 (2002). 